\title{
Bilateral Peroneal Tendon Subluxation of an Adolescent Female with MRI Correlations
}

\author{
Daniel R Barr, MSc ${ }^{1 *}$, Nicholas L Newcomb, BS ${ }^{1}$ and McCall G McDaniel, MD ${ }^{2}$ \\ ${ }^{1}$ University of Queensland-Ochsner Clinical School, New Orleans, Louisiana, USA \\ ${ }^{2}$ Ochsner Medical Center, New Orleans, Louisiana, USA
}

*Corresponding author: Daniel R Barr, MSc, University of Queensland-Ochsner Clinical School, 1315 Jefferson Hwy, New Orleans, LA 70121, USA

\begin{abstract}
Background: Peroneal subluxation is relatively rare and commonly misdiagnosed as an ankle sprain. Due to its rarity, the incidence of subluxation is unknown, but it has been seen most often in young adults. Peroneal subluxation in pediatric patients is very rare and bilateral subluxation is even rarer. We present a pediatric case of bilateral peroneal tendon subluxation caused by minimal trauma with no known risk factors. Based on a literature review, this is the first reported case of acquired bilateral peroneal tendon subluxation in a pediatric patient. We illustrate the patient presentation, MRI correlations, operative descriptions, and outcomes.
\end{abstract}

Case description: A 14-year-old female competitive cheerleader and gymnast presented with bilateral ankle pain 17 days after a training injury. She had fallen off of a tightrope and both of her feet rotated inward, becoming caught in the rope. Since then, she noticed bilateral subluxation of her distal peroneus tendons. Bilateral ankle MRIs demonstrated a large amount of fluid signal intensity around the peroneal tendons consistent with tendinitis and subluxation. The patient did not improve after 6 weeks in walking casts and physical therapy and underwent bilateral operative superior retinacular repair. Four months post operation, she was able to return to gymnastics and cheerleading with minimal difficulty.

Conclusions: Bilateral SPR repair was found to be effective in this very rare pediatric case of acquired bilateral peroneal tendon subluxation. In similar presentations we recommend consideration be given to this treatment course.

\section{Keywords}

Peroneal tendon, Subluxation, Tendinitis, Pediatric, Retinacular repair

\section{Introduction}

The peroneal muscles of the leg provide dynamic stabilization of the ankle joint with the distal tendon running through the retro-malleolar groove on the fibula to insert onto the inferior aspect of the metatarsal bones. The superior peroneal retinaculum (SPR) is a fibrous band that helps to hold the peroneal tendons in place and act as a primary restraint to prevent subluxation of the tendons. Laxity of the SPR, lateral ankle instability, or a groove with insufficient depth may increase the risk of subluxation [1]. Additionally, recent cortisone or fluoroquinolone therapy has been shown to increase risk of tendon rupture which can present with subluxation [2]. Peroneal subluxation is relatively rare and is commonly misdiagnosed as an ankle sprain. Due to its rarity, the incidence of subluxation is unknown, but it has been seen most often in young adults. However, peroneal tears which can be related to subluxations have been found in $11-37 \%$ of cadaveric samples [3].

Sudden and forceful contraction of the peroneal muscles while the foot is dorsiflexed is the primary mechanism of subluxation of the peroneal tendons and it is usually unilateral, affecting only the side of injury. Swelling and tenderness can be found posterior to the lateral malleolus. The tendons may subluxate anteriorly and click when the ankle is rotated or dorsiflexed and everted [4]. Surgical intervention remains the preferred method of treating symptomatic cases of subluxation. Multiple procedures can be utilized to repair the tendon; however, the specific procedure is often decided upon 
intraoperatively depending on what is found. The body of the SPR may need to be repaired, it may need to be reattached to its insertion point, or the retro-malleolar groove may need to be deepened [5]. The surgery may be complicated by the presence of open fibular growth plates; care must be taken to not interrupt these growth plates in pediatric patients [6]. Peroneal subluxation in pediatric patients with open physes is uncommon with the first case of surgical correction of this condition in a pediatric patient with open physes being reported in 2000 [7].

We report a pediatric case of bilateral peroneal tendon subluxation caused by minimal bilateral trauma with no known risk factors. This study aims to demonstrate the effectiveness of bilateral SPR repair procedures.

\section{Case Description \\ Presentation and imaging}

A 14-year-old female competitive cheerleader and gymnast presented with bilateral ankle pain 17 days after a training injury. She had slipped off of a tightrope and as she fell, both of her feet rotated inward, becoming caught in the rope. Immediately after the fall, she was taken to an urgent care where bilateral ankle radiographs were taken. The imaging was unremarkable, and she was told her ankles were sprained. Her ankles were wrapped, and she was advised to limit weight
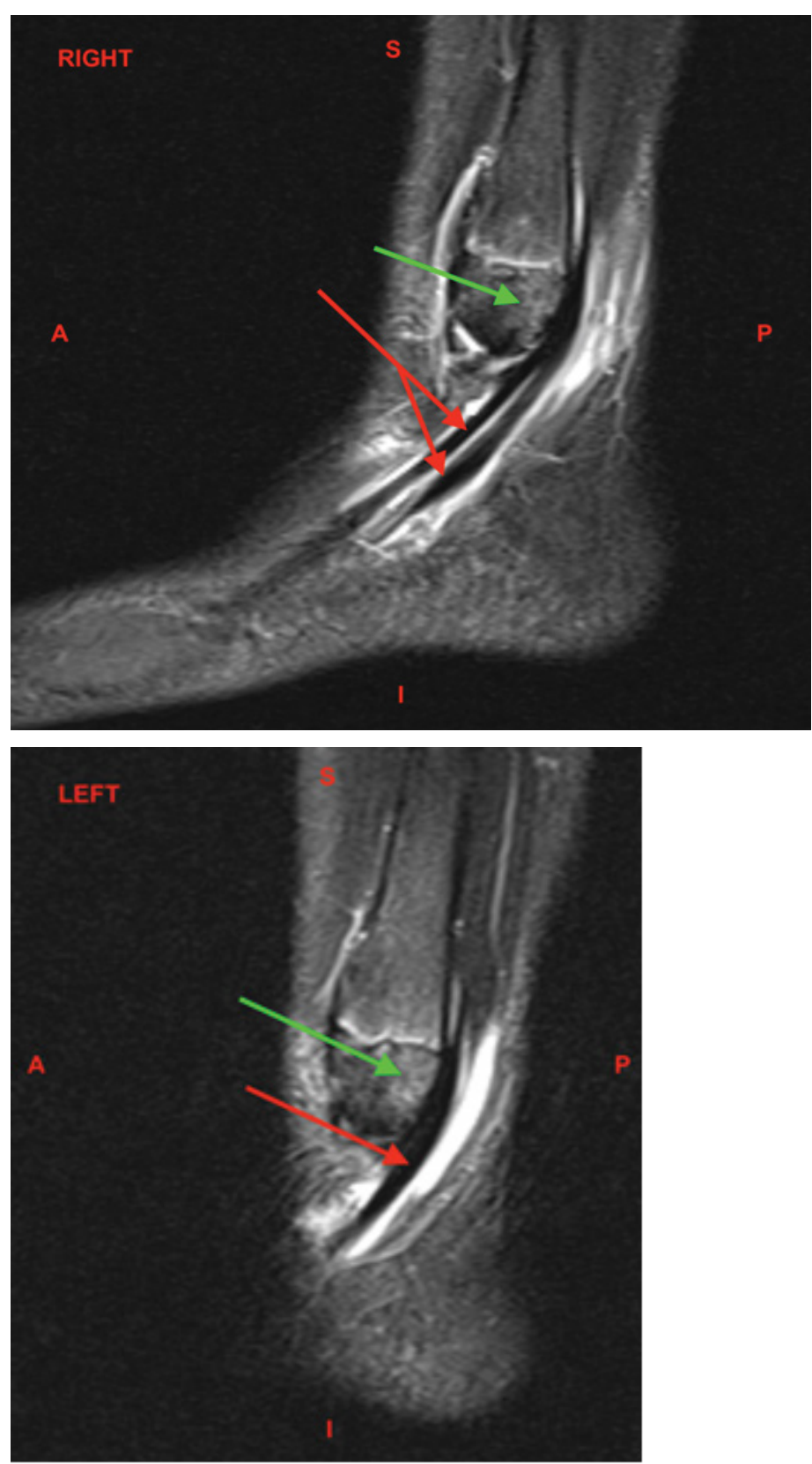

Figures 1 and Figure 2: MRI Ankle, Bilateral, Sagittal, T2 Fat Suppressed without contrast. The red arrows demonstrate the large amount of fluid signal intensity about the peroneus longus and brevis tendons indicative of tenosynovitis. The green arrows demonstrate the bone marrow edema within the lateral distal aspect of the lateral malleolus, likely reactive to the peroneus tendon subluxation.

S: Superior; I: Inferior; A: Anterior; P: Posterior 
bearing activity for two weeks. Since then, her ankles continued to hurt and swell. She described a popping sensation over the back of her distal fibula bilaterally and explained that she had to manually "pop her tendons back behind the bone." She had no prior ankle injuries and her past medical history was only significant for a prior nondisplaced distal radius fracture. Review of systems was negative for additional abnormalities.

On examination, the patient had an antalgic gait. Both ankles were swollen without extensive erythema. There was edema and ecchymosis around the peroneal tendons which were tender to palpation. No subluxation of the peroneal tendons was reproduced with manipulation. The patient was not tender over the anterior talofibular ligament (ATFL), calcaneofibular ligament (CFL), deltoid ligament, or distal fibula bilaterally. The flexor hallucis longus (FHL), extensor hallicus longus (EHL), tibialis anterior muscles were intact. The patient had decreased ankle range of motion bilaterally, and pain over the peroneus tendons with plantarflexion and eversion. The patient was neurovascularly intact bilaterally (Figure 1 and Figure 2).

The patient was placed in bilateral controlled ankle movement (CAM) walker boots and ankle MRIs were completed 10 days after orthopedic consultation. The ankle MRIs were nearly identical bilaterally. Both demonstrated moderate to large amounts of fluid signal intensity around the peroneus longus and brevis tendons compatible with tenosynovitis. Minimal peroneus longus tendinosis was seen at the distal margin of the lateral malleolus bilaterally. Bone marrow edema was seen within the lateral distal aspect of the lateral malleolus, reactive from the peroneal tendon pathology. Soft tissue edema was seen surrounding the area. No injuries were seen to the articular cartilage. The extensor tendons, tibialis posterior, flexor digitorum longus (FDL), flexor hallicus longus (FHL), and Achilles tendon appeared intact. The anterior and posterior tibiofibular, talofibular, calcaneofibular, and deltoid ligaments appeared intact (Figure 3 and Figure 4).

\section{Intervention and outcomes}

The patient was diagnosed with bilateral peroneal subluxation with resulting tendinitis. She was placed in bilateral walking casts which were worn for six weeks. There were no instances of subluxation while in the casts. After 6 weeks, the patient was placed back in CAM walking boots and began physical therapy. During physical therapy sessions, however, she had developed recurrent subluxation of the left peroneal tendons. There were no instances of right sided subluxation.

After having failed conservative therapy, the patient underwent left superior peroneal retinacular repair. On operative inspection, the superficial peroneal retinaculum had partially avulsed off of the lateral malleolus with no tears found in the tendon. Three holes were then drilled into the distal fibula, mindfully avoiding the growth plate. Groove deepening was not performed as to not disturb the patient's growth plates. FiberWire sutures were applied to the posterior aspect of the superior peroneal retinaculum through the holes in the fibula and tied down, with the anterior portion of the retinaculum pulled over the repair and sutured to the posterior aspect of the superficial peroneal retinaculum, with the peroneal tendons moving freely in the new tightened retinaculum after being secured. The patient was placed in a short-leg cast in slight inversion and plantar flexion to assist in keeping the tendons in the groove and remained non-weight bearing on the left foot.

Seven days post operation, the patient developed painful recurrent subluxation of the right (non-operative) peroneal tendon. She described that weight bearing exclusively on the right extremity post-operation was now aggravating the right ankle. Now with recurrent right sided symptoms, the patient opted to undergo a second operative superior retinacular repair on the right side. Similar to the left side, the right superficial peroneal retinaculum had partially avulsed off of the lateral malleolus without tendinous tearing. Four holes were drilled into the distal fibula, again avoiding the growth plate and again groove deepening wasn't performed to avoid injuring the growth plates. FiberWire sutures were used to tie the superior retinaculum down to the distal fibula in the same fashion as the contralateral side. The peroneal tendons moved freely in the newly tightened retinaculum after being secured.

Post-operatively, the patient was placed in a second short-leg cast and remained wheelchair bound for 6 weeks with bilateral lower extremity casts. She then transitioned to bilateral CAM walker boots and started to bear weight on her left leg but remained nonweightbearing on the right for an additional two weeks. The patient underwent weekly physiotherapy and was able to bear weight as tolerated. Four months postoperations, she was able to return to gymnastics and cheerleading where she was able to complete a backflip with minimal difficulty.

\section{Conclusions}

Bilateral SPR repair procedures were found to be effective in this pediatric case of acquired bilateral peroneal tendon subluxation following a bilateral trauma with no known risk factors. Within the pediatric population, there is limited literature surrounding this injury. The majority of studies involve adult patients and yet anterior subluxation of the peroneal tendon is still considered to be an uncommon condition [8]. Furthermore, this injury is typically unilateral, with bilateral cases being linked to congenital conditions [9]. To the best of our knowledge, this is the first reported case of acquired bilateral peroneal tendon subluxation in a pediatric patient. 

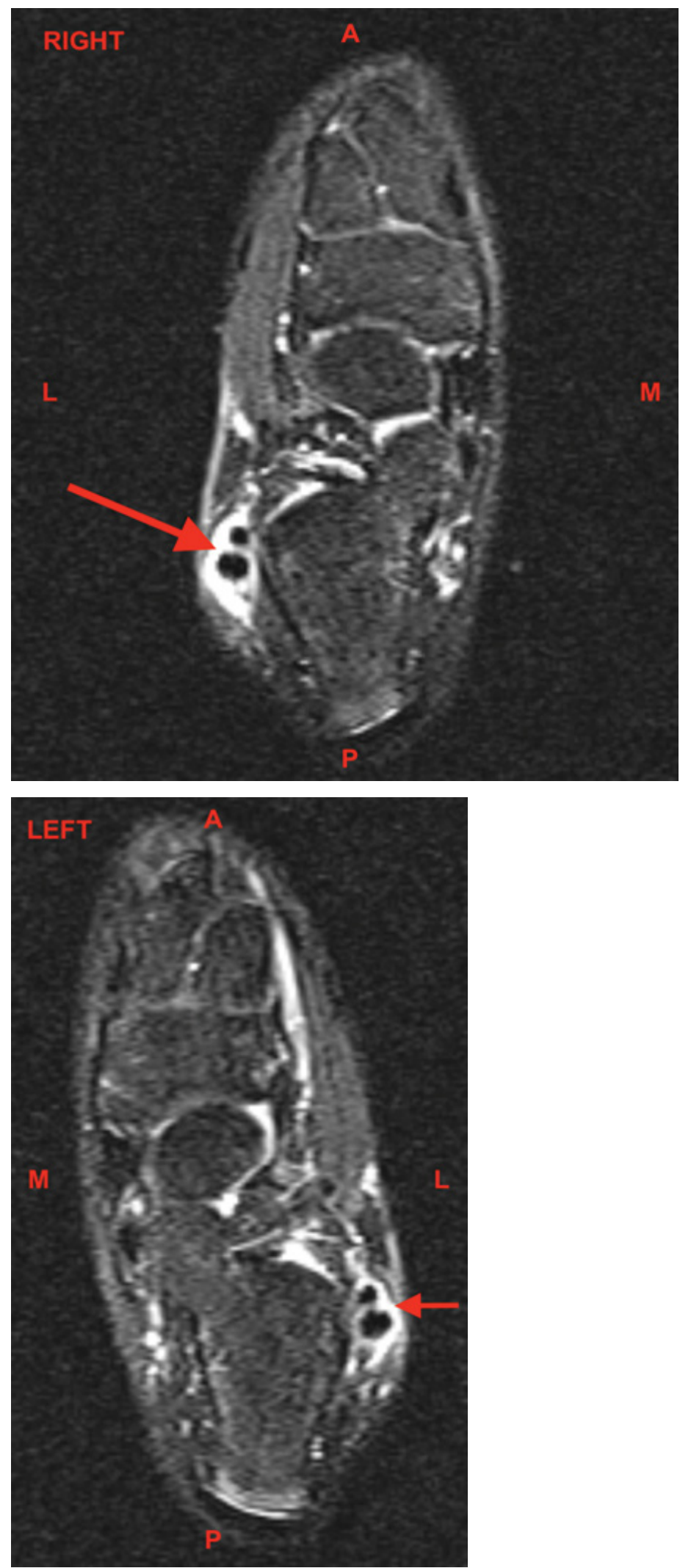

Figures 3 and Figure 4: MRI Ankle, Bilateral, Sagittal, T2 Fat Suppressed without contrast. The red arrows demonstrate the large amount of fluid signal intensity about the peroneus longus and brevis tendons indicative of tenosynovitis.

A: Anterior; P: Posterior; M: Medial; L: Lateral

These injuries are often initially handled with conservative treatment, such as taping or compressive bandages, however conservative treatment alone has an overall failure rate of $50-76 \%$ for adults, with no reported literature on pediatric patients [10]. The most successful non-operative intervention is a below-theknee plaster cast for 4-6 weeks which has prevented recurrent dislocation in $62-83 \%$ of adults reviewed [11]. If conservative treatment fails, various surgical techniques can be considered with an overall recurrence rate of less 
than $1.5 \%$ at long-term follow-up. Adult patients that are treated surgically with both SPR repair and with peroneal tendon groove deepening have a higher rate of returning to sports when compared to patients that only received an SPR repair [12]. In this case, conservative therapy on the left ankle failed to prevent subluxation leading to surgical intervention. Casting was initially successful on the right side, but eventually led to recurrence once this side started to bear full body weight. The patient opted to complete a second operative intervention 14 days after the first, requiring her to be nonambulatory and wheelchair-bound for four weeks. A groove deepening procedure was not performed due to the risk to her active growth plates.

Overall, the treatment course was highly successful with a full return to high intensity gymnastics and cheerleading, with the patient even able to complete a backflip only 4 months post-operation. In similar presentations we recommend consideration be given to this treatment course.

\section{References}

1. Selmani E, Gjata V, Gjika E (2006) Current concepts review: Peroneal tendon disorders. Foot Ankle Int 27: 221-228.

2. Scheidegger P, Weisskopf L, Hirschmüller A (2017) Atraumatic bilateral rupture of the peroneus brevis tendon in recreational sport: A case report. SAGE Open Med Case Rep 5.
3. Squires N, Myerson MS, Gamba C (2007) Surgical treatment of peroneal tendon tears. Foot Ankle Clin 12: 675-695.

4. Oesman I, Kurniawan D, Wikanjaya R (2019) Peroneal groove deepening as the treatment of peroneal tendon subluxation: A case report. Int J Surg Case Rep 65: 333338.

5. Saragas NP, Ferrao PNF, Mayet Z, Eshraghi H (2016) Peroneal tendon dislocation/subluxation-Case series and review of the literature. Foot Ankle Surg 22: 125-130.

6. Jaglowski JR, Boykin R, Kocher M (2012) Recurrent peroneal tendon subluxation in children and adolescents. Current Orthopaedic Practice 23: 104-107.

7. Forman ES, Micheli LJ, Backe LM (2000) Chronic recurrent subluxation of the peroneal tendons in a pediatric patient. Surgical recommendations. Foot Ankle Int 21: 51-53.

8. Cohen I, Lane S, Koning W (1983) Peroneal tendon dislocation: A review of the literature. J Foot Surg 22: 15-20.

9. Rivard DS, Sobiesk G, Wertheimer SJ (1998) Bilateral congenital peroneal tendon subluxation. J Foot Ankle Surg 37: 339-344.

10. Ferran NA, Oliva F, Maffulli N (2006) Recurrent subluxation of the peroneal tendons. Sports Med 36: 839-846.

11. Bakker D, Schulte JB, Meuffels DE, Piscaer TM (2019) Non-operative treatment of peroneal tendon dislocations: A systematic review. J Orthop 18: 255-260.

12. van Dijk PAD, Gianakos AL, Kerkhoffs GMMJ, Kennedy JG (2016) Return to sports and clinical outcomes in patients treated for peroneal tendon dislocation: A systematic review. Knee Surg Sports Traumatol Arthrosc 24: 1155-1164. 